Research Article

\title{
Routing Algorithm with Uneven Clustering for Energy Heterogeneous Wireless Sensor Networks
}

\author{
Ying Zhang, ${ }^{1}$ Wei Xiong, ${ }^{1}$ Dezhi Han, ${ }^{1}$ Wei Chen, ${ }^{2}$ and Jun Wang ${ }^{1,3}$ \\ ${ }^{1}$ College of Information Engineering, Shanghai Maritime University, Shanghai 201306, China \\ ${ }^{2}$ Department of Computer Science, Tennessee State University, Nashville, TN 37209, USA \\ ${ }^{3}$ Department of Electrical and Computer Engineering, University of Central Florida, Orlando, FL 32816, USA
}

Correspondence should be addressed to Wei Xiong; xiongwei501@163.com, Dezhi Han; dzhan@shmtu.edu.cn, and Wei Chen; wchen@tnstate.edu

Received 3 June 2016; Accepted 14 August 2016

Academic Editor: Fei Yu

Copyright ( 2016 Ying Zhang et al. This is an open access article distributed under the Creative Commons Attribution License, which permits unrestricted use, distribution, and reproduction in any medium, provided the original work is properly cited.

\begin{abstract}
Aiming at the "hotspots" problem in energy heterogeneous wireless sensor networks, a routing algorithm of heterogeneous sensor network with multilevel energies based on uneven clustering is proposed. In this algorithm, the energy heterogeneity of the nodes is fully reflected in the mechanism of cluster-heads' election. It optimizes the competition radius of the cluster-heads according to the residual energy of the nodes. This kind of uneven clustering prolongs the lifetime of the cluster-heads with lower residual energies or near the sink nodes. In data transmission stage, the hybrid multihop transmission mode is adopted, and the next-hop routing election fully takes account of the factors of residual energies and the distances among the nodes. The simulation results show that the introduction of an uneven clustering mechanism and the optimization of competition radius of the cluster-heads significantly prolonged the lifetime of the network and improved the efficiency of data transmission.
\end{abstract}

\section{Introduction}

With the extension of detecting areas by human beings, information obtaining, information storage, and information processing, transmission, and application are embedded to every aspect in social production and daily life. Wireless sensor networks (WSNs) [1] integrated with the frontier technologies, such as sensing, microelectromechanical systems (MEMS), wireless communication, and distributed information processing, have emerged as the time requires. A wireless sensor network is comprised of a large number of small and cheap sensor nodes. The nodes communicate with each other by agreement strategies, and the communication strategies are administered by routing protocols [2]. The wireless sensor network has a wide application prospect in real life. It has an important research value and huge practicality value in many fields: environment monitoring, rescue and relief work, remote control in hazardous area, and so on. It will be recommended and applied in many fields with great favor $[3,4]$.
Nowadays, with the development of marine economy, the huge economic benefit is being brought about from the exploitation of marine resources, and meanwhile this causes environmental disruption in many marine areas. There are frequent occurrences of ocean disasters. How to dynamically detect the environmental factors becomes a serious challenge. With the development of sensors and computer networking, WSNs play an important role in the development of marine environment monitoring and forecasting [5-7]. Sensor nodes deployed in the ocean will change their initial position owing to the effect of ocean currents, and the AUVs (Autonomous Underwater Vehicles) collect the underwater environmental information in moving state. These all cause the dynamical change of the network's topology.

Normally, the nodes of a wireless sensor network have limited energies. Due to the one-off deployment, it is difficult to replenish the energies [8]. Due to the different duty and equipping for the sensor nodes, the energy consumption will be different among them. So, it is an energy heterogeneous sensor network. Sometimes, the so-called "hotspots" easily 
occur in sensor networks with heterogeneous energies in practical application. It is important to find an optimized route for the information transmission of the network. Routing protocols $[9,10]$ will be responsible for discovering and maintaining energy efficient routes and make communication reliable and efficient. "Hotspots" will make some nodes come to an untimely end due to the excessive energy consumption, and this will shorten the life cycle of the whole network [11]. Therefore, it is necessary to design the energy efficient protocol to adapt the features of this kind of sensor network. For the unbalanced energy consumption of the sensor networks, cluster-based sensor networks enable achieving efficient utilization of the limited energy resources of the deployed sensor nodes. The uneven clustering is suitable to be used in heterogeneous sensor networks [12]. In this way, it can extend the lifetime of the networks the furthest. The cluster-based routing protocol, whose goal is to reduce the node's energy consumption and improve the network lifetime, has become one of the hot topics in the study of wireless sensor networks.

The rest of this article is organized as follows. In Section 2, some related works on cluster-based routing algorithm for sensor networks are introduced. In Section 3, some necessary models are defined. Then, in Section 4, we describe the EDEUC method in detail. Section 5 comprises the simulation and results analysis, and finally we conclude the article in Section 6.

\section{Related Work}

According to the sensory ability, computing power, communication capacity, and carrying of energies, sensor nodes can be divided into different levels. Heterogeneous sensor networks mean a network composed of a variety of different types of sensor nodes [13]. The form organized into a cluster of sensor nodes can effectively reduce the network energy consumption. Some energy efficient routing protocols are designed based on the cluster structure. Currently, many cluster algorithms, such as LEACH [14, 15], PEGASIS [16], and HEED [17], are all set up based on homogeneous network. However, the pure homogeneous network is inexistent in practical application. It is just an ideal assumption. Each kind of protocol has its own characters. The algorithms used in homogeneous network cannot be duplicated to be used in heterogeneous network directly, so it is necessary to design the clustered-based routing algorithm which is suitable to be used in heterogeneous network $[18,19]$.

In recent years, many literatures have studied the feasibility of heterogeneous sensor networks in practice application, and some clustering methods for heterogeneous sensor networks were proposed. Solar LEACH [20] was proposed in 2004 by Voigt et al., which is an improved protocol based on extending the ordinary LEACH to two class level heterogeneous sensor networks. EELBC algorithm [21] was proposed by Jayashree et al., who structured the two-class clustering network model with cluster-head based on heterogeneous nodes; this protocol optimizes the deployment of the ordinary sensor nodes and the cluster-head nodes, so it can extend the life cycle of the network. SEP protocol [22] was proposed in 2004 by Smaragdakis et al. In this protocol, nodes are divided into two kinds of nodes: normal nodes and advanced nodes. The advanced nodes have more chances to become the cluster-head, but this method only considers one factor: residual energy for the choice of cluster-head. Moreover, this method chooses one hop transmission mode for data transferring in inner cluster or among the clusters. The literature [23] proposed DEEC algorithm aiming at ordinary multilevel heterogeneous wireless sensor networks. The cluster-head can be elected based on residual energies of the nodes and the average energy of the network, and it can make the nodes with high initial energy and residual energy have more chances to become the cluster-head, so the energy consumption of the network could be balanced, and the cycle time of the network is prolonged. Cai et al. [24] proposed the RCR (Reliable Clustering Routing) method. It establishes a predictive model of residual energy, REP (Remaining Energy Prediction) applied to multilevel energies heterogeneous network, and it can solve the problem of energy loss for single node due to some kinds of fault. Every cluster will elect multicluster-heads to afford the tasks of cluster-heads jointly, but it has the problem of only considering one factor in the cluster election. Wang et al. [25] proposed the cluster method of load balance, LBCA (Load Balance Clustering Algorithm), which can be applied in multilevel energies heterogeneous sensor networks. It can elect the cluster-heads according to the energies distribution in the detecting area. The nodes with lower average communication energy have high priority to become the cluster-head in the area with homogeneous energy distribution, and the nodes with higher residual energies will have high priority to become the cluster-head in the area with unbalanced distribution of energy. Aiming at the problem of lifetime for heterogeneous network, the literature [26] proposed an optimal algorithm of lifetime for multilevel energies heterogeneous sensor networks: SNLOC (Sensor Network Lifetime Optimization Clustering Algorithm). The literature [27] proposed an uneven cluster-based routing protocol for wireless sensor networks. It makes the nodes near the sink node have a small scale cluster by the uneven competition scope, and the unequal-sized scope is determined by the subjective settings. So, they all do not give a practical algorithm to realize the uneven clustering for this kind of sensor network systematically and effectively.

In this paper, we proposed a routing algorithm for multilevel energies heterogeneous sensor networks based on uneven clustering, EDEUC (Energy-Distance Efficiency Based on Uneven Clustering), which constructs an optimal path to the base station with multihops by cluster-heads. The EDEUC strategy can reduce the energy consumption of the nodes and prolong the life cycle of sensor networks effectively.

The main contribution of the article reflects as follows: the energy heterogeneity is fully considered in the election of cluster-heads, the competition radius of the cluster-heads is optimized according to the residual energy of the nodes, and this improves the stability of clusters. The lifetime of the nodes with lower residual energies or near the sink nodes can be prolonged by this kind of uneven clustering mechanism and this improves the stability of the networks. The hybrid 
multihop transmission mode is adopted in data transmission stage. The next-hop routing election fully takes account of the factors of residual energies and the distances among the nodes, and this improves the efficiency of data transmission.

\section{Network Model}

3.1. Assumption of Network Model. For the heterogeneous network model, we suppose that there are $N$ sensor nodes distributed in a two-dimensional square area: $M \times M$ randomly. The nodes collect environment information periodically. The sensor network has features as follows:

(1) The sensor network is the static network, and the nodes can only have micromotion or hold still after deployment.

(2) The energy of the base station is controllable. The energy is heterogeneous for all the nodes, and the energies cannot be supplemented.

(3) All the nodes have the function of data fusion, and every node has a unique ID.

(4) The communication between the nodes in cluster and cluster-head adopts single hop mode, and the communication between cluster-head and the sink node adopts multihop mode by relay.

The wireless channel is symmetrical. The energy consumption for transmitting one bit from node $A$ to node $B$ is equal to the energy consumption for transmitting one bit from node $B$ to node $A$.

3.2. Energy Model for Sensor Networks. In multilevel energy heterogeneous network, the initial energy of nodes is distributed randomly in the closed interval: $\left[E_{0}, E_{0}\left(1+\alpha_{i}\right)\right]$, where $E_{0}$ is the minimum energy of the nodes and $\alpha_{i} \in(0,1)$ is the multiple coefficient of the initial energy of node $S_{i}$ more than $E_{0}$. Suppose the number of all the nodes in the network is $N$. The initial energy of the whole multilevel energies heterogeneous network can be presented as the following formula:

$$
E_{\text {total }}=\sum_{i=1}^{N} E_{0}\left(1+\alpha_{i}\right)=E_{0}\left(N+\sum_{i=1}^{N} \alpha_{i}\right) .
$$

The calculation of energy loss in the process of wireless communication is in accordance with the energy consumption model in the literature [21]. Suppose the threshold of communication distance $d_{0}=\sqrt{\varepsilon_{\mathrm{fs}} / \varepsilon_{\mathrm{mp}}}$; when the distance of nodes transmitting data $d<d_{0}$, the channel model of free space will be used; when the distance of nodes transmitting data $d \geq d_{0}$, the multichannel attenuation model will be used as well.

The energy consumption of sensor node transmitting the data of $k$ bit can be described as

$$
\begin{aligned}
E_{\mathrm{Tx}}(k, d) & =E_{\mathrm{Txelec}}(k)+E_{\mathrm{Txamp}}(k, d) \\
& = \begin{cases}k E_{\mathrm{elec}}+k \varepsilon_{\mathrm{fs}} d^{2}, & d<d_{0} \\
k E_{\mathrm{elec}}+k \varepsilon_{\mathrm{fs}} d^{4}, & d \geq d_{0} .\end{cases}
\end{aligned}
$$

The energy consumption of sensor node receiving the data of 1 bit can be described as

$$
E_{\mathrm{Rx}}(k)=E_{\text {Rxelec }}(k)=k E_{\text {elec }} .
$$

The model includes the energy consumption of transmitting data and receiving data. The consumption of transmitting data includes the energy consumption of radiofrequency circuit and signal amplifier. The consumption of receiving nodes is the energy consumption of receiving circuit. Where $\varepsilon_{\mathrm{fs}}$ and $\varepsilon_{\mathrm{mp}}$ are the magnifications of amplifier, $E_{\text {elec }}$ denotes the energy consumption of transmitting or receiving 1-bit data.

\section{EDEUC Algorithm}

The core idea of the algorithm is the mechanism of uneven clustering routing which is introduced into the sensor network with heterogeneous energy. The energy factor is introduced to the competition radius of cluster-head, so it can fully make use of the property of heterogeneous energy of the nodes. Before electing the cluster-head of the nodes, the number of the competition cluster-heads can be restrained by electing the candidates of cluster-head, and then the final nodes of cluster-head will be elected from these candidate cluster-heads. The next routing nodes can be chosen by considering energies of nodes and the transmitting distances in the process of data transferring comprehensively.

4.1. Election of Candidate Cluster-Heads. In the time of network running, all the nodes in the network will participate in the competition to become the candidate cluster-heads. Every node $S_{i}(i=1,2, \ldots, N)$ will produce a random number $\mu \in(0,1)$. If $\mu$ is less than the threshold $T\left(S_{i}\right)$, the relevant node will be the candidate cluster-head, and it is expressed as formula (4). In order to fully make use of the property of heterogeneous energy of nodes, the factor of residual energy of node $S_{i}$ is introduced into weighting probability of the competition of cluster-heads. It is expressed as formula (5). $k_{\text {opt }}$ is introduced into threshold $T\left(S_{i}\right)$ for candidate cluster-heads in the election mechanism. $k_{\text {opt }}$ is the optimal cluster number of the network: $k_{\text {opt }}=\sqrt{N / 2 \pi}$. $\sqrt{\varepsilon_{\mathrm{fs}} / \varepsilon_{\mathrm{mp}}} \cdot\left(M / d_{\mathrm{toBS}}^{2}\right)$, where $d_{\mathrm{toBS}}$ is the distance between the node $S_{i}$ and base station. This will cause the threshold to become larger, and it will ensure that more nodes have the opportunity to become the candidate cluster-heads. The node sets of candidate cluster-head can be denoted as $S_{i} \cdot S_{\mathrm{ch}}$ :

$$
\begin{aligned}
& S_{i} \cdot S_{\mathrm{ch}}=\left\{S_{j} \text { is the candidate cluster-head } \mid S_{j} \cdot \mu\right. \\
& \left.\quad \in(0,1)<T\left(S_{j}\right)\right\} .
\end{aligned}
$$

$S_{j} \cdot \mu$ is the random number produced by node $S_{j}$. $T\left(S_{j}\right)$ is the threshold of candidate cluster-head of node $S_{j}$ accordingly. The average probability for node $S_{i}$ becoming cluster-head can be calculated as in the following formula:

$$
p\left(S_{i}\right)=\frac{p_{\mathrm{opt}} \cdot N \cdot\left(1+\alpha_{i}\right)}{N+\sum_{i=1}^{N} \alpha_{i}} \cdot \frac{E_{i}(r)}{E_{\mathrm{ave}}(r)},
$$


where $p_{\text {opt }}=k_{\text {opt }} / N$ is the ratio of the optimal cluster-head number for the network, $E_{i}(r)$ is the residual energy of the node currently, and $E_{\text {ave }}(r)$ is the average residual energy for all the nodes of the network in the $r$ round currently. In this algorithm, the threshold $T\left(S_{i}\right)$ is denoted as in the following formula:

$$
\begin{aligned}
& T\left(S_{i}\right) \\
& = \begin{cases}\frac{p\left(S_{i}\right)}{1-p\left(S_{i}\right) \cdot\left(r \cdot \bmod \left(1 / p\left(S_{i}\right)\right)\right)} \cdot k_{\mathrm{opt}}, & S_{i} \in G \\
0, & \text { the other, }\end{cases}
\end{aligned}
$$

where $p\left(S_{i}\right)$ is the probability of the node becoming the candidate cluster-head, $r$ is the round currently, $r \cdot \bmod \left(1 / p\left(S_{i}\right)\right)$ is the number of the nodes to ever become the candidate cluster-head in this round, and $G$ is the set of the nodes not to become the candidate cluster-head in this round.

4.2. Competition Radius of Candidate Cluster-Head. In order to achieve uneven clustering in the network, the method of designing different competition radius of candidate clusterhead is adopted. However, this traditional method only considers the distance factor among the nodes in the design of cluster-head competition radius, and the energy factor is not considered in the process. For the sensor network with heterogeneous energy, the node with higher residual energy should have larger communication radius, so the energy of nodes could be balanced like this. In order to solve the problem of "hotspots" in sensor network, the factors of energy and distance should be considered comprehensively in the calculation of cluster-head radius. The candidate cluster-heads calculate the cluster-head radius by formula (7) according to their residual energy and the distance between themselves and the base station.

$$
\begin{aligned}
& R_{c_{i}} \\
& \quad=\left[1-p_{1}\left(\frac{d_{\max }-d\left(c_{i}, \mathrm{BS}\right)}{d_{\max }-d_{\min }}\right)-p_{2}\left(1-\frac{E_{c_{i}}}{E_{\text {ave }}}\right)\right] R_{c},
\end{aligned}
$$

where $p_{1}$ and $p_{2}$ are the regulative parameters of distance and energy, respectively, and they are in the range of $(0,1)$. $d_{\max }$ is the farthest distance from the candidate cluster-head to the base station, and $d_{\min }$ is the shortest distance from the candidate cluster-head to the base station. $E_{\text {ave }}$ is the average residual energy of all the candidate cluster-heads. $E_{c_{i}}$ is the residual energy of the candidate cluster-head currently. $R_{c}$ is the maximal competition radius of the candidate clusterheads. $d\left(c_{i}, \mathrm{BS}\right)$ is the distance from candidate cluster-head $c_{i}$ to base station. From formula (7), we know that the greater the residual energy of the candidate cluster-heads is, the larger the competition radius of the cluster-heads will be. The closer the distance from the candidate cluster-heads to the base station is, the smaller the competition radius of candidate cluster-head will be.

4.3. Election of Cluster-Head. After electing the candidate cluster-heads, these candidate cluster-heads will broadcast the information (COMPETE_HEAD_MSG) of competing cluster-head in the range of their radius of cluster-head competition. The content of the information includes the node ID and the current residual energy $E_{i}$. The candidate clusterheads begin to compete with the cluster-head by different starting time $\left(T_{c_{i}}\right)$. Energy factor is mainly considered in this kind of election system. If a candidate cluster-head has a shorter starting time to elect the cluster-head, this means it will have higher priority to become the cluster-head. If the time at which the candidate cluster-head starts to elect is longer than the latest time of starting to elect the clusterhead preset by the system, the candidate cluster-head will not be the cluster-head. The time $\left(T_{c_{i}}\right)$ at which the candidate cluster-head starts to elect the cluster-head can be calculated as follows:

$$
T_{c_{i}}=\frac{E_{\mathrm{ave}}\left(E_{\max }-E_{i}\right)}{E_{i}\left(E_{\max }-E_{\mathrm{ave}}\right)} \cdot T_{c},
$$

where $E_{\max }$ is the largest initial energy of the candidate cluster-head, $E_{i}$ is the current residual energy of the node, and $T_{c}$ is the latest time of starting to elect cluster-head preset by the system.

4.4. Data Transmission Mode. After setting up the cluster, it should enter the phase of data transferring. The mixed multihop transferring mode should be adopted between clusterhead and base station. The transmission mode of multihop may share the energy consumption with multiclusters rather than concentrating on one cluster. It can balance the energy consumption of clusters effectively. Suppose that the clusterhead $c_{i}$ indirectly sends data to base station via the relay cluster-head $c_{j}$; the set of routing nodes where the clusterhead $c_{i}$ will choose the next hop can be expressed as $c_{i} \cdot R_{\mathrm{ch}}$ :

$$
\begin{aligned}
c_{i} \cdot R_{\mathrm{ch}} & =\left\{c_{j} \mid d^{2}\left(c_{j}, \mathrm{BS}\right) \leq d^{2}\left(c_{i}, \mathrm{BS}\right)\right. \\
& \left.\cup\left(d^{2}\left(c_{j}, \mathrm{BS}\right)+d^{2}\left(c_{i}, c_{j}\right)\right)<d^{2}\left(c_{i}, \mathrm{BS}\right)\right\},
\end{aligned}
$$

where $d\left(c_{i}, \mathrm{BS}\right)$ is the distance from the cluster-head $c_{i}$ to base station BS, $d\left(c_{j}, \mathrm{BS}\right)$ is the distance from relay clusterhead $c_{j}$ to base station, and $d\left(c_{i}, c_{j}\right)$ is the distance from the cluster-head $c_{i}$ to the relay cluster-head $c_{j}$. If the set $c_{i} \cdot R_{\mathrm{ch}}$ is an empty set, this indicates that the cluster-head $c_{i}$ has no appropriate relay routing nodes for the next hop, and the cluster-head $c_{i}$ will directly communicate with the base station. If the set $c_{i} \cdot R_{\mathrm{ch}}$ is not an empty set, this means that there exist relay routing nodes for the next hop. The clusterhead $c_{i}$ will choose the appropriate relay routing node from the set according to the parameter weight $W_{j}$. The residual energy of the nodes and distance among the cluster-heads will be considered comprehensively in this process.

Definition 1. The distance factor $D_{c_{i} \text { BS }}$ is introduced to the mechanism of choosing the routing nodes for the next hop by cluster-head $c_{i}$. It can be described as in the following formula:

$$
D_{c_{i} \text { BS }}=\left(d^{2}\left(c_{i}, c_{j}\right)+d^{2}\left(c_{j}, \mathrm{BS}\right)\right) \times e^{-E_{c_{j}} / E_{\text {ave }}},
$$


where $E_{c_{j}}$ is the current residual energy of relay cluster-head $c_{j}$ and $E_{\text {ave }}$ is the current average residual energy of the whole network.

In the mechanism of choosing the relay cluster-heads, the distance factor $D_{c_{i} \text { BS }}$ will mainly consider two aspects: energy and distance; that is to say, the path and energy consumption for sending data by cluster-head $c_{i}$ should be considered comprehensively. If we only consider the distance factor for the next-hop routing, we cannot elect the appropriate routing path roundly. The energy factor of all the cluster-heads should be considered sufficiently in this process.

Definition 2. The energy balance factor $m_{c_{i}}$ is introduced to the process of choosing relay cluster-head $c_{j}$ by the clusterhead $c_{i}$ according to its own residual energy. The value of $m_{c_{i}}$ can be expressed as in the following formula:

$$
m_{c_{i}}= \begin{cases}d^{2}\left(c_{i}, c_{j}\right), & E_{c_{i}} \leq E_{c_{j} \text { ave }} \\ 0, & E_{c_{i}}>E_{c_{j} \text { ave }}\end{cases}
$$

where $E_{c_{j} \text { ave }}$ is the average residual energy of candidate relay cluster-head $c_{j}$.

From formula (11), we know that when the residual energy of current cluster-head $c_{i}$ is larger than the average residual energy of candidate relay cluster-head $c_{j}$, we shall not worry too much about the problem of excessive energy consumption of cluster-head $c_{i}$. On the contrary, when the surplus energy of cluster-head $c_{i}$ is smaller, the factor of distance between cluster-heads $c_{i}$ and $c_{j}$ should be fully considered in the election of relay cluster-head. Therefore, in order to reduce the energy consumption of the source clusterhead, the transmission distance $d^{2}\left(c_{i}, c_{j}\right)$ between clusterheads $c_{i}$ and $c_{j}$ can be introduced to restrain the election of relay cluster-head.

Definition 3. The energy balance factor $m_{c_{j}}$ is introduced to the process of electing the relay cluster-head by the candidate relay cluster-head $c_{j}$ according to its own residual energy. The value of $m_{c_{j}}$ can be expressed as in the following formula:

$$
m_{c_{j}}= \begin{cases}K, & E_{c_{j}} \leq E_{c_{j} \text { ave }} \\ 0, & E_{c_{j}}>E_{c_{j} \text { ave }},\end{cases}
$$

where $K$ is a constant. From formula (12), we know that when the residual energy of relay cluster-head $c_{j}$ is larger, the cluster-head will take on more responsibility in the process of forwarding data. It can avoid the energy being exhausted with taking on the task of forwarding data for the cluster-heads with less residual energy. The energy balance factor $m_{c_{j}}$ is just introduced to constrain the cluster-heads with lower energy to become the next-hop routing nodes.

Definition 4. On the base of Definition 1 to Definition 3, the parameter weight $w_{j}$ is introduced to the process of choosing the relay cluster-head $c_{j}$ from the cluster-head $c_{i}$. This weight
TABLE 1: Simulation parameters.

\begin{tabular}{lccc}
\hline Parameters & Values & Parameters & Values \\
\hline$E_{\text {elec }}$ & $50 \mathrm{~nJ} / \mathrm{bit}$ & $\varepsilon_{\mathrm{fs}}$ & $10 \mathrm{pJ} / \mathrm{bit} / \mathrm{m}^{2}$ \\
$E_{\mathrm{DA}}$ & $5 \mathrm{~nJ} / \mathrm{bit} / \mathrm{message}$ & $\varepsilon_{\mathrm{mp}}$ & $0.0013 \mathrm{pJ} / \mathrm{bit} / \mathrm{m}^{4}$ \\
$E_{0}$ & $0.5 \mathrm{~J}$ & Message size & $40000 \mathrm{bits}$ \\
$p$ & 0.1 & $n$ & 100 \\
\hline
\end{tabular}

considers the relationship of distance factor and energy factor comprehensively. It can be expressed as in the following formula:

$$
w_{j}=p D_{i_{-} \mathrm{BS}}+q\left(m_{c_{i}}+m_{c_{j}}\right),
$$

where $D_{i \text { BS }}$ is the factor of distance from the cluster-head $c_{i}$ to base station, $m_{c_{i}}$ is the energy balance factor of the clusterhead $c_{i}, m_{c_{j}}$ is the residual energy balance factor of the relay cluster-head $c_{j}, p$ and $q$ are the weight regulation parameters, and $p+q=1$.

The cluster-head $c_{i}$ can calculate the weight $w_{j}$ of candidate relay cluster-heads in the set of $c_{i} \cdot R_{\mathrm{ch}}$ by formula (13). The next-hop relay routing nodes can be chosen in the relay cluster-heads according to the size of the weights. The election law of relay cluster-head is that the smallest of the parameter weights $w_{j}$ of the cluster-head will be the next-hop routing nodes. In order to reduce the energy consumption of storing information in the node, there are only 3 pieces of routing information stored in the routing table of cluster-head $c_{i}$. The routing information in the routing table is stored according to the sequence from the small weight to the large weight. The cluster-head with the smallest weight will be the first routing choice.

\section{Simulation Results and Performance Analysis}

5.1. Simulation Environment. In order to test and verify the energy saving performance and balance performance of energy loss, EDEUC was compared with some widely used algorithms: SEP and DEEC in this experiment. The simulation environment settings are as follows: supposing 100 nodes with heterogeneous energies are distributed in the square area $((x=0, y=0),(x=100, y=100))$ randomly. The base station locates in the point of $(x=$ $50, y=150$ ). Suppose $m$, which is the proportion of the nodes with advanced energies to all the nodes, is 0.5 . In the heterogeneous network, $E_{0}=0.5 \mathrm{~J}, \alpha_{\max }=1$, the nodes of the sensor network are distributed in the closed interval $\left[E_{0},\left(1+E_{0}\right)\right]$ randomly, so we can get the initial energy interval of the nodes: $[0.5,1]$. The other parameters of the simulation environment are listed in Table 1.

$p$ is the proportion of optimizing clusters, $E_{\mathrm{DA}}$ is the energy consumption of data fusion by cluster-head, and $n$ is the number of the nodes. These parameters above should be used in the evaluation of energy consumption. Besides these, there are other parameters that need to be configured; 


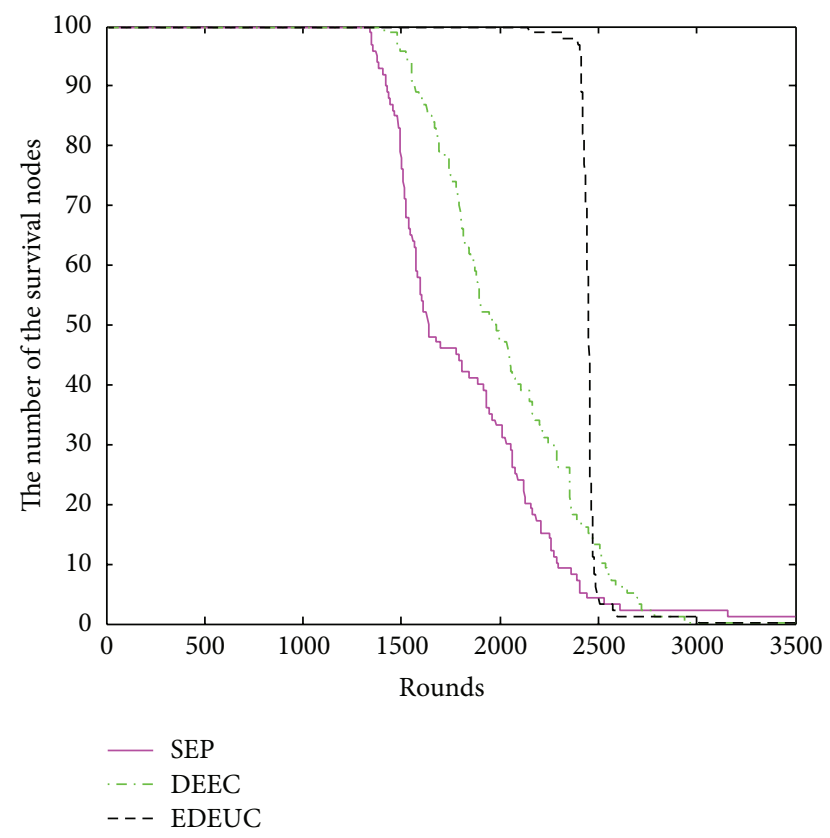

FIgURE 1: The number of survival nodes changes with rounds updating.

for example, $p_{1}$ and $p_{2}$ can be used to control the size of competition radius of cluster-head and $R_{c}$ is the largest competition radius of cluster-head. After lots of testing and simulation, the values can be set up as follows: $R_{c}=60 \mathrm{~m}$, $p_{1}=0.3$, and $p_{2}=0.2$. The regulation parameters of distance and energy in the multihop transmission mechanism are set up as $p=0.6$ and $q=0.4$.

5.2. Experimental Results and Analysis. The network life cycle is an important index of the performance evaluation for a routing algorithm. Figure 1 indicates the changes of number of the live nodes in the network with rounds updating.

Figure 1 illustrates that the first dead node in EDEUC algorithm can last more rounds than others, so this indicates that EDEUC algorithm has a longer stable life cycle than the others. The first dead node of SEP, DEEC, and EDEUC has died in the round of 1444,1586 , and 2256, respectively. DEEC algorithm has improved by $9.8 \%$ compared to DEEC, and EDEUC has improved by $42.2 \%$ compared to DEEC. EDEUC has a relative small time span while the network is dying, which is because EDEUC algorithm considers the factors of the residual energy of nodes and the distance of communication, and the proportionality of energy consumption is better than the others. Due to the introduction of the mechanism of uneven clustering to the EDEUC algorithm, the clusterhead can fully make use of the feature of heterogeneous energy of the nodes in the stage of cluster-head structuring and data transferring. It can balance the energy consumption of cluster-head and extend the life cycle of the network effectively.

Figure 2 illustrates that different value assigned to the parameter weight of competition radius of cluster-head will influence the life cycle. When $\left(p_{1}, p_{2}\right)$ is assigned with

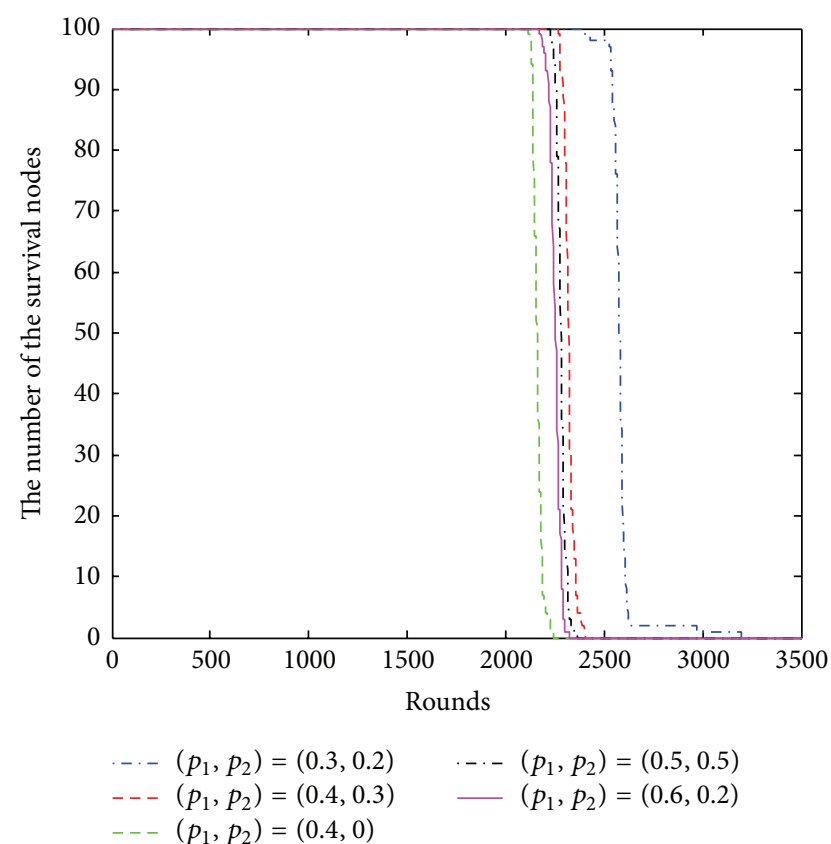

Figure 2: Different value assigned to $p_{1}$ and $p_{2}$ influences the life cycle of the network.

$(0.4,0)$, this means we only consider the communication distance of the nodes in the process of determining the competition radius of cluster-head and ignore the factor of residual energies. The other assignments of the parameters will optimize the situation of choosing competition radius of cluster-head. Figure 2 illustrates that the life cycle of network will be the shortest one if we do not optimize the competition radius of cluster-head. That is to say, the communication distance and the residual energy of the node should be both considered when we determine the competition radius of cluster-head. When $\left(p_{1}, p_{2}\right)$ is with the value of $(0.3,0.2)$, the life cycle of the network will be the longest compared to the other cases. In other cases for $\left(p_{1}, p_{2}\right)$, life cycles of the network have relative closer results. So it is reasonable that $\left(p_{1}, p_{2}\right)$ should be chosen as $(0.3,0.2)$ in the several groups of data above.

EDECU method adopts the hybrid multihop transmission mechanism. It can reduce the energy consumption of data transmission for cluster-heads. In order to verify the advantages of energy consumption of cluster-heads, we chose 10 rounds of data randomly in the process of network running and compare the results of energy consumption with DEEC and SEP methods. Figure 3 illustrates the volume of energy consumption of cluster-heads for these three kinds of methods.

Figure 3 illustrates that the energy consumption of cluster-head by using EDEUC method is obviously lower than the energy consumption of cluster-head by using DEEC and SEP methods, and the energy consumption of clusterhead by using SEP method is the largest compared to others. EDEUC method improves two aspects, choice of clusterhead and data transmission, and its multihop transmission mechanism can effectively reduce energy consumption of 


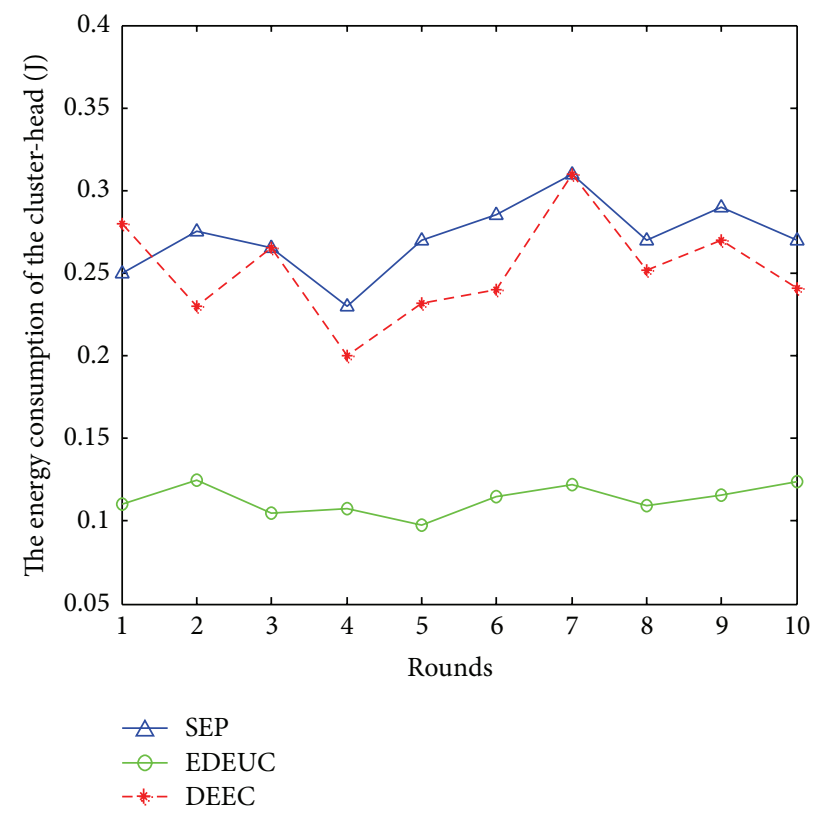

FIgURE 3: The sum of the energy consumption of cluster-heads.

cluster-heads. EDEUC is proposed as a kind of uneven clustering routing algorithm based on the characters of nodes with heterogeneous energies. The residual energy and the distance of transmission data of the nodes are both considered comprehensively in the mechanism of choosing cluster-head and data transferring, so the distribution of cluster-head nodes is more reasonable. This can effectively prevent the occurring of network "hotspots." The energy consumption of cluster-head by using EDEUC algorithm has better performance than the other two algorithms.

Figure 4 illustrates the analysis results of the algorithm performance from the view of residual energy of the network. The residual energy of network can impliedly indicate the performance of life cycle and the energy consumption of the network. The more residual energy the network has, the less the energy consumption of the network is and the longer the life cycle of the network will be.

Figure 4 also illustrates that the residual energy of the network by using SEP or DEEC algorithm is less than by using EDEUC algorithm in most time stages. Along with the network operation to late stage (after 1000 rounds), the residual energy of network by using EDEUC algorithm is more than the others. So the performance of the two aspects regarding the node energy utilization and the balance of network's energy consumption is better than the other two algorithms.

Figure 5 illustrates the data volume of network transferring by these three algorithms. It is clear that not only does EDEUC algorithm have better performance of the balance of network energy consumption than the others, but also it can have the network transfer more data volumes to base station. Lower energy consumption of network is in favor of prolonging the lifetime of the network, so the network could keep sending more data in the longer period of time, and

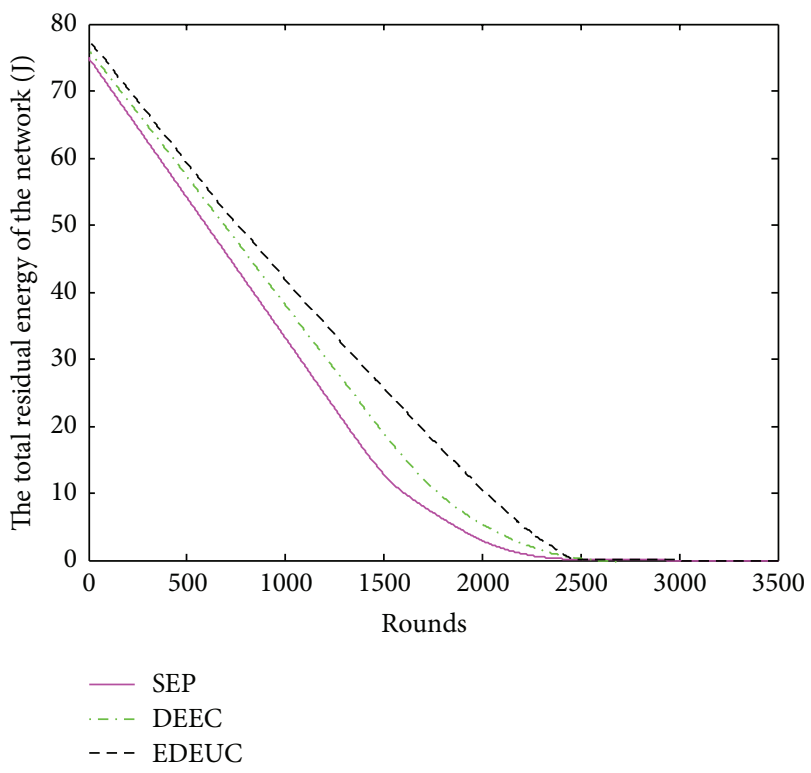

FIgURE 4: The residual energy of the network changes over time.

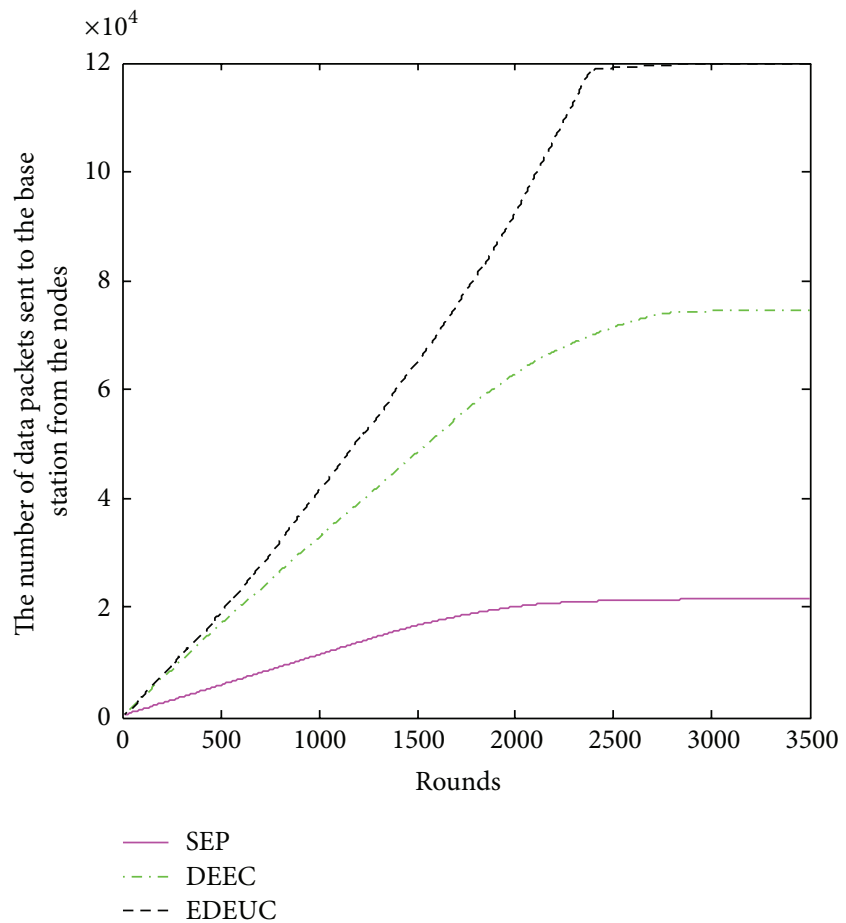

FIGURE 5: The amount of data received by base station with rounds updating.

the amount of data received by base station will be bound to increase.

In order to clearly observe the amount of data transferred by using these different algorithms, we choose four stages of network running time to compare them to each other in the experiments. In the horizontal ordinate of Figure 6, the four stages are the round of $1000,1500,2000$, and 2500, respectively. We can compare these total data volumes of the four 


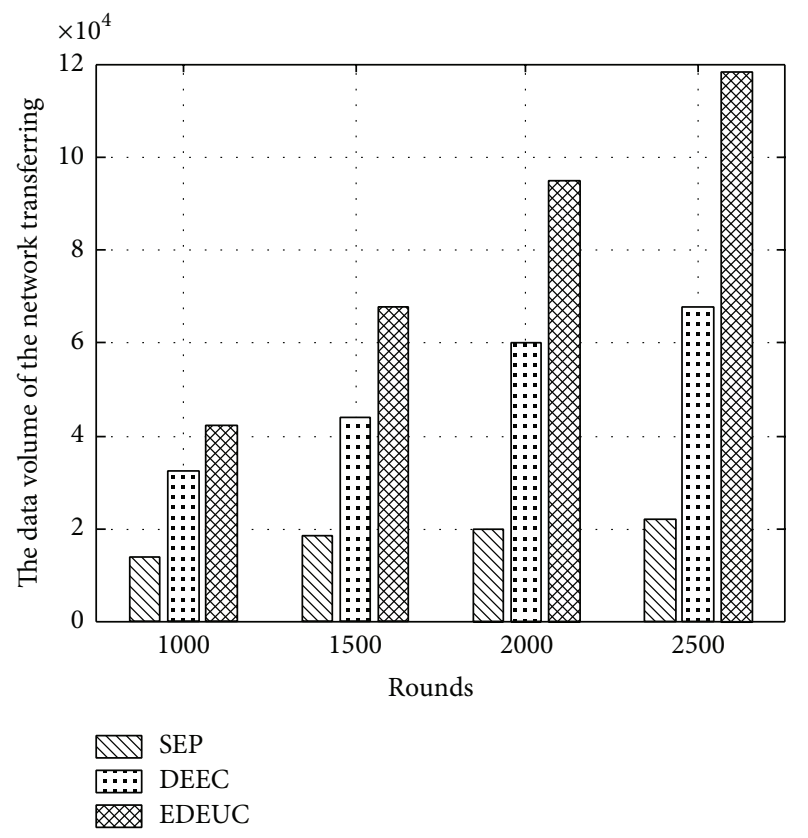

FIGURE 6: Columnar contrast figure of the amount of data transferred by network with different algorithms.

states as columnar contrast figure visually in Figure 6 . When the network running time reaches 2000 rounds, the network can transfer more data volumes by using EDEUC algorithm, and it can improve by $380 \%$ and $75 \%$ compared with SEP and DEEC algorithms, respectively. After 2000 rounds, the data volume transferred by SEP algorithm increases less, and the total data volume transferred by network tends to have stable status. But, for the other two algorithms, the data volume transferred still increases, and obviously the data volume transferred by using EDEUC algorithm increases more than the others.

\section{Conclusion}

In order to solve the problem of "hotspots" in sensor networks, a kind of routing algorithm named EDEUC based on energies and distances was proposed by using the idea of uneven clustering. This method adopts double selection mechanism for cluster-heads and optimizes the competition radius of cluster-heads. The hybrid multihop transmission is adopted in the stage of data transferring. The simulation result indicates that this kind of uneven clustering mechanism and the optimizing for competition radius of clusterheads can obviously improve the transmission performance of the network and enhance the life cycle of the network effectively.

\section{Competing Interests}

The authors declare that they have no competing interests.

\section{Acknowledgments}

This work was supported by the National Natural Science Foundation of China (nos. 61273068, 61673259, and 61373028) and the International Exchanges and Cooperation Projects of Shanghai Science and Technology Committee (no. 15220721800).

\section{References}

[1] A. A. Abbasi and M. Younis, "A survey on clustering algorithms for wireless sensor networks," Computer Communications, vol. 30, no. 14-15, pp. 2826-2841, 2007.

[2] S. P. Singh and S. C. Sharma, "A survey on cluster based routing protocols in wireless sensor networks," Procedia Computer Science, vol. 45, pp. 687-695, 2015.

[3] A. N. Knaian, "A wireless sensor network for smart roadbeds and intelligent transportation systems," Tech. Rep., Electrical Science and Engineering, Massachusetts Institute of Technology, 2000.

[4] Y. Liang and R. Liu, "Routing topology inference for wireless sensor networks," ACM SIGCOMM Computer Communication Review, vol. 43, no. 1, pp. 21-28, 2013.

[5] Z. Zhou, B. Yao, R. Xing, L. Shu, and S. Bu, "E-CARP: an energy efficient routing protocol for UWSNs in the internet of underwater things," IEEE Sensors Journal, vol. 16, no. 11, pp. 4072-4082, 2015.

[6] N. Javaid, M. R. Jafri, Z. A. Khan, N. Alrajeh, M. Imran, and A. Vasilakos, "Chain-Based communication in cylindrical underwater wireless sensor networks," Sensors, vol. 15, no. 2, pp. 3625-3649, 2015.

[7] I. Nevat, G. W. Peters, F. Septier, and T. Matsui, "Estimation of spatially correlated random fields in heterogeneous wireless sensor networks," IEEE Transactions on Signal Processing, vol. 63, no. 10, pp. 2597-2609, 2015.

[8] S. Dehghani, M. Pourzaferani, and B. Barekatain, "Comparison on energy-efficient cluster based routing algorithms in wireless sensor network," Procedia Computer Science, vol. 72, pp. 535$542,2015$.

[9] A. Chamam and S. Pierre, "A distributed energy-efficient clustering protocol for wireless sensor networks," Computers and Electrical Engineering, vol. 36, no. 2, pp. 303-312, 2010.

[10] M. Azharuddin, P. Kuila, and P. K. Jana, "Energy efficient fault tolerant clustering and routing algorithms for wireless sensor networks," Computers \& Electrical Engineering, vol. 41, pp. 177190, 2015.

[11] S. Soro and W. B. Heinzelman, "Prolonging the lifetime of wireless sensor networks via unequal clustering," in Proceedings of the 19th IEEE International Parallel and Distributed Processing Symposium (IPDPS '05), pp. 1-8, Denver, Colo, USA, April 2005.

[12] T. C. Karalar, S. Yamashita, M. Sheets, and J. Rabaey, "A low power localization architecture and system for wireless sensor networks," in Proceedings of the IEEE Workshop on Signal Processing Systems Design and Implementation, pp. 8994, Piscataway, NJ, USA, October 2004.

[13] H. Xia, R.-H. Zhang, J. Yu, and Z.-K. Pan, "Energy-efficient routing algorithm based on unequal clustering and connected graph in wireless sensor networks," International Journal of Wireless Information Networks, vol. 23, no. 2, pp. 141-150, 2016. 
[14] W. Heinzelman, A. Chandrakasan, and H. Balakrishman, "Energy-efficient communication protocol for wireless microsensor networks," in Proceedings of the 33rd Hawaii International Conference on System Science, pp. 3005-3014, Maui, Hawaii, USA, January 2000.

[15] S. Tyagi and N. Kumar, "A systematic review on clustering and routing techniques based upon LEACH protocol for wireless sensor networks," Journal of Network and Computer Applications, vol. 36, no. 2, pp. 623-645, 2013.

[16] S. Lindsey and C. S. Raghavendra, "PEGASIS: power-efficient gathering in sensor information systems," in Proceedings of the IEEE Aerospace Conference, pp. 1125-1130, March 2002.

[17] O. Younis and S. Fahmy, "HEED: a hybrid, energy-efficient, distributed clustering approach for ad hoc sensor networks," IEEE Transactions on Mobile Computing, vol. 3, no. 4, pp. 366379, 2004.

[18] B. A. Attea and E. A. Khalil, "A new evolutionary based routing protocol for clustered heterogeneous wireless sensor networks," Applied Soft Computing Journal, vol. 12, no. 7, pp. 1950-1957, 2012.

[19] J. Yu, Y. Qi, G. Wang, and X. Gu, "A cluster-based routing protocol for wireless sensor networks with nonuniform node distribution," AEU-International Journal of Electronics and Communications, vol. 66, no. 1, pp. 54-61, 2012.

[20] T. Voigt, A. Dunkels, J. Alonso et al., "Solar-aware clustering in wireless sensor networks," in Proceedings of the 9th International Symposium on Computers and Communications (ISCC '04), vol. 1, pp. 238-243, July 2004.

[21] L. S. Jayashree, S. Arumugam, and N. Rajathi, "E/sup 2/LBC: an energy efficient load balanced clustering technique for heterogeneous wireless sensor networks," in Proceedings of the IFIP International Conference on Wireless and Optical Communications Networks, pp. 1-7, IEEE, Bangalore, India, April 2006.

[22] G. Smaragdakis, I. Matta, and A. Bestavros, SEP: A Stable Election Protocol for Clustered Heterogeneous Wireless Sensor Networks, Computer Science Department, Boston University, Boston, Mass, USA, 2004.

[23] L. Qing, Q. Zhu, and A. Wang, "A distributed energy-efficient clustering algorithm for heterogeneous wireless sensor networks," Journal of Software, vol. 17, no. 3, pp. 481-489, 2006.

[24] H.-B. Cai, X.-M. Ju, and Q.-Y. Cao, "Energy prediction and reliable clustering routing protocol for multilevel energy heterogeneous wireless sensor networks," Chinese Journal of Computers, vol. 32, no. 12, pp. 2393-2402, 2009.

[25] X. Wang, G. Zhang, and X. Xie, "A load balance clustering algorithm for multilevel energy heterogeneous wireless sensor networks," Journal of Computer Research and Development, vol. 45, no. 3, pp. 392-399, 2008.

[26] Y. Liang, "An optimized clustering algorithm for energy heterogeneous wireless sensor networks," Transactions of Shenyang Ligong University, vol. 28, no. 2, pp. 57-61, 2009.

[27] C.-F. Li, G.-H. Chen, M. Ye, and J. Wu, "Uneven cluster-based routing protocol for wireless sensor networks," Chinese Journal of Computers, vol. 30, no. 1, pp. 27-36, 2007. 


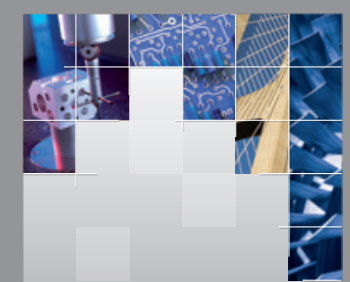

\section{Enfincering}
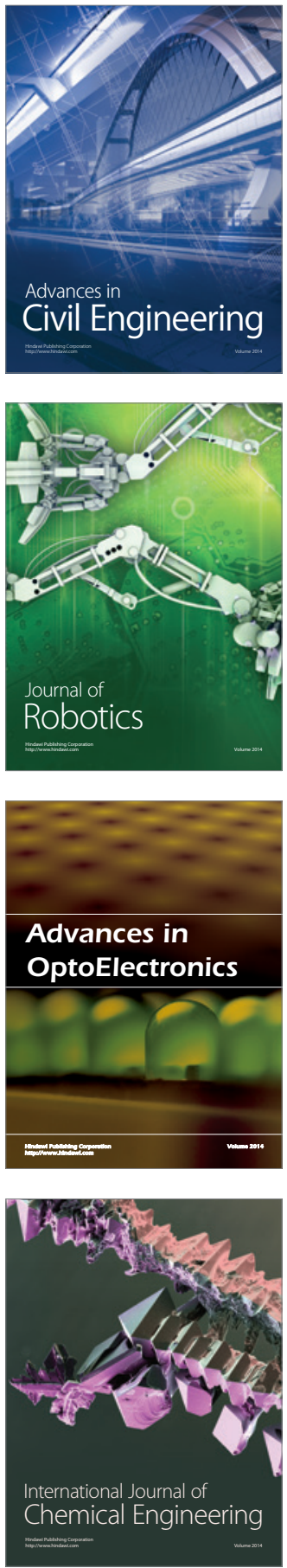

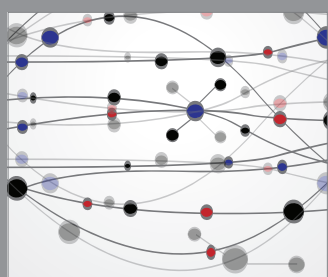

The Scientific World Journal

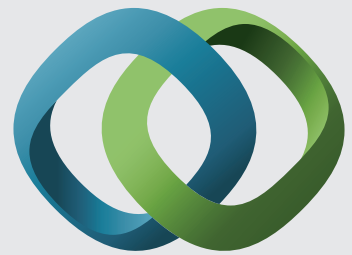

\section{Hindawi}

Submit your manuscripts at

http://www.hindawi.com
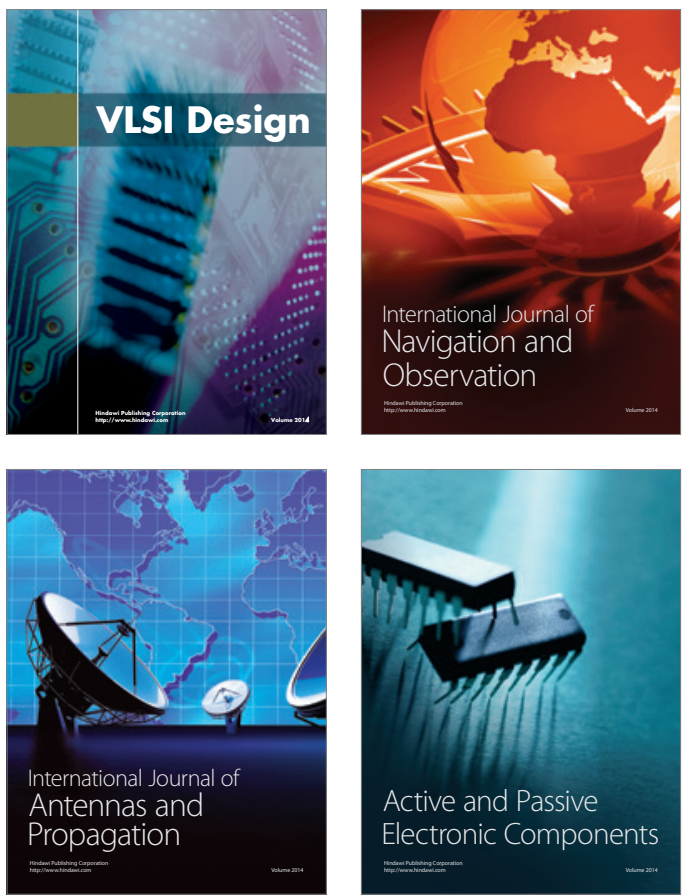
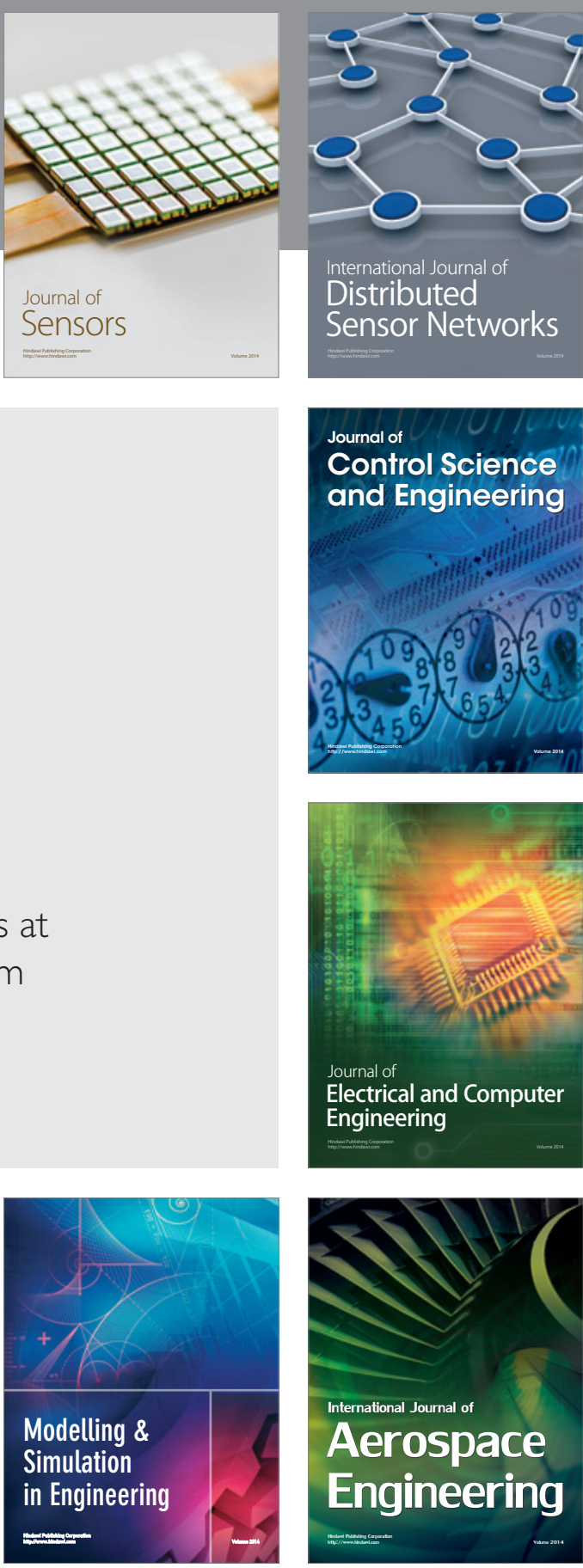

International Journal of

Distributed

Sensor Networks

Journal of

Control Science

and Engineering
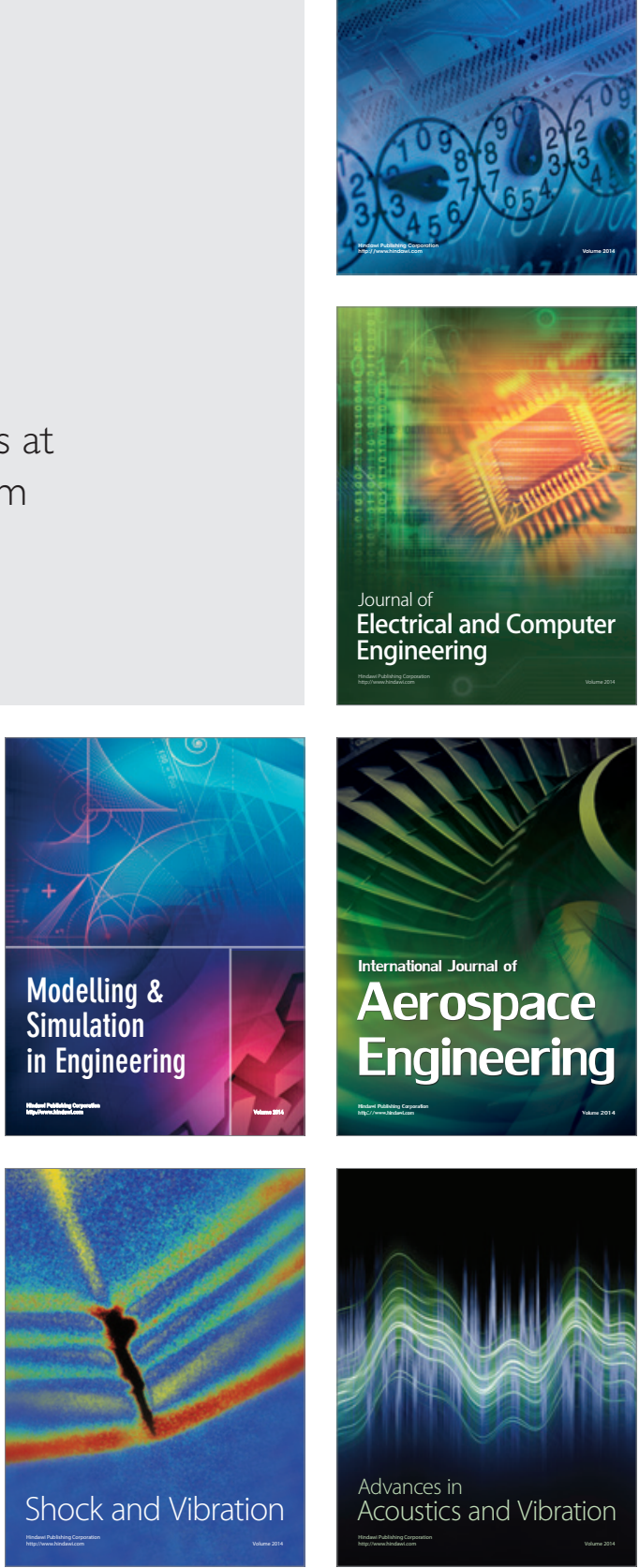